\title{
Biological activities of Pereskia bleo extracts
}

\begin{abstract}
The aim of this study is to screen the hexane, dicholoromethane, ethyl acetate and methanol extracts of Pereskia bleo (PB) for their antibacterial, anti-oxidant and anti-cancer properties using disc diffusion method, DPPH assay and MTT cytotoxicity test, respectively. It is found that hexane and methanol extracts showed highly and moderately, respectively, considerable antibacterial activity towards two Gram-negative bacteria, P. aeruginosa 60690 and S. choleraesuis. Ethyl acetate extract showed a week narrow spectrum activity $(=35 \%$ of streptomycin activity). The highest antibacterial activity on MRSA is obtained by DCM extract. Hexane extract was the most effective DPPH radical scavenger (37.55\%). Ethyl acetate and DCM extracts were less effective free radical scavenger $(16.1 \%)$. None of the extracts were cytotoxic significantly towards MCF-7, HT-29 and CEM-SS cell lines after 72 $\mathrm{h}$ incubation time (IC50>30 $\mathrm{g} \mathrm{mL}-1$ ). It could be concluded that antibacterial activity of P. bleo is the most promising biological activity attributed to this plant.
\end{abstract}

Keyword: Anti-oxidant; Anti-tumor; Antibacterial; Pereskia bleo 